Check for updates

Cite this: RSC Adv., 2018, 8, 31967

\title{
Fluorescent polycatecholamine nanostructures as a versatile probe for multiphase systems $\uparrow$
}

\author{
Vinayaraj Ozhukil Kollath, (1) \$ Maziar Derakhshandeh, \$ Francis D. Mayer, \\ Thanmayee Mudigonda, Muhammad Naoshad Islam, Milana Trifkovic \\ and Kunal Karan (iD *
}

Shape and size controlled nanostructures are critical for nanotechnology and have versatile applications in understanding interfacial phenomena of various multi-phase systems. Facile synthesis of fluorescent nanostructures remains a challenge from conventional precursors. In this study, bio-inspired catecholamines, dopamine (DA), epinephrine (EP) and levodopa (LDA), were used as precursors and fluorescent nanostructures were synthesized via a simple one pot method in a water-alcohol mixture under alkaline conditions. DA and EP formed fluorescent spheres and petal shaped structures respectively over a broad spectrum excitation wavelength, whereas LDA did not form any particular structure. However, the polyepinephrine (PEP) micropetals were formed by weaker interactions as compared to covalently linked polydopamine (PDA) nanospheres, as revealed by NMR studies. Application of these fluorescent structures was illustrated by their adsorption behavior at the oil/water interface using laser scanning confocal microscopy. Interestingly, PDA nanospheres showed complete coverage of the oil/water interface despite its hydrophilic nature, as compared to hydrophobic PEP micropetals which showed a transient coverage of the oil/water interface but mainly self-aggregated in the water phase. The reported unique fluorescent organic structures will play a key role in understanding various multi-phase systems used in aerospace, biomedical, electronics and energy applications.

Received 22nd June 2018
Accepted 17th August 2018

DOI: $10.1039 / \mathrm{c} 8 \mathrm{ra05372c}$

rsc.li/rsc-advances the system. The use of shape and size controlled nanoparticles can increase the fundamental understanding of such multiphase systems, which can then be employed to further improve the properties of such systems. Simulations have shown that particle shape can have a dramatic effect on both the interfacial packing structure and equilibrium phase behavior of materials. ${ }^{12,13}$ When used in combination with other interactions and functionalization schemes, particle shape can play a key role in the design of materials for self-assembly.

Dopamine (DA), belongs to the catecholamine group of molecules better known for their mussel-inspired chemistry and neurotransmitter behaviour and PDA nanostructures formed of it can be spherical shape if produced in a controlled water-alcohol-alkaline medium. However, little is known about the nanostructures formed from other molecules of the same class such as epinephrine (EP) and 3,4-dihydroxy-L-phenylalanine (levodopa; LDA) (see Fig. 1a for chemical structure). It is expected that these molecules may yield different shape and surface charge because of the numerous factors at interplay in the formation of the nanostructures as is now understood for PDA. ${ }^{14}$ The PDA, or dopamine-melanin, nanoparticles formation can be considered to be a two-step process: formation of oligomers from covalent linkages between the monomeric free radical units, followed by self-assembly of oligomers via noncovalent interactions. ${ }^{14-17}$ The variation in PDA NP size is
Department of Chemical and Petroleum Engineering, The University of Calgary, 2500 University Drive N.W., Calgary, Alberta T2N 1N4, Canada.E-mail: kkaran@ucalgary. $\mathrm{ca}$

$\dagger$ Electronic supplementary information (ESI) available: Experimental details and extended results mentioned in the main document. See DOI: 10.1039/c8ra05372c \$ Authors V. O. K. and M. D. contributed equally to this work. 

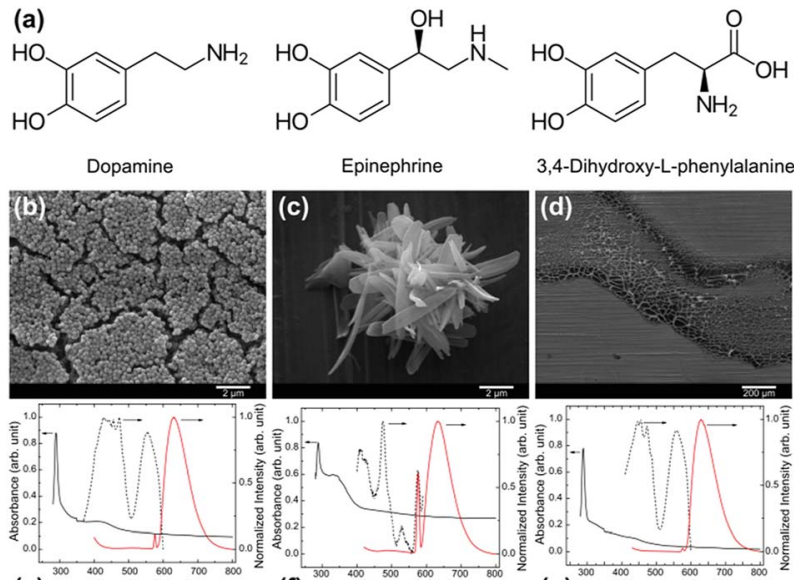

(e)

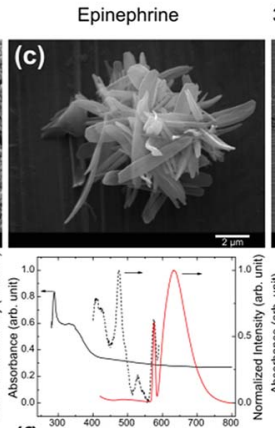

3,4-Dihydroxy-L-phenylalanine
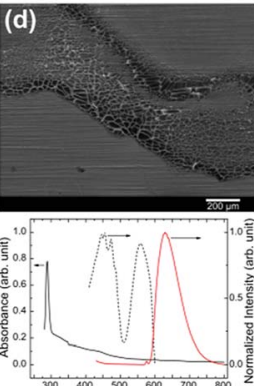

(g)

aggregation state is. We also observe quantitative differences in the rheology of emulsions stabilized by nanoparticles with different precursors, which we attribute to the shape-mediated interactions coupled with the difference in their affinity for the interface.

Spherical nanoparticle formation from carbon precursors in water-alcohol mixture under alkaline condition has been related to the Stöber method so far. ${ }^{20,21}$ Inspired by the nanospheres produced from DA, we report the outcome of similar catecholamine molecules under similar conditions. The results of this study indicate that the spherical shape formation cannot be generalized for all catecholamine molecules. The structure of the synthesized PDA, PEP, and LDA particles examined by SEM are shown in Fig. 1b-d. The PDA particles are spherical with approximately $200 \mathrm{~nm}$ diameter (Fig. 1b). The shape and size of these particles were reproducible. A few earlier studies reported PDA spheres with similar size and shape uniformity. ${ }^{6,22-24}$ The EP precursor yields a petal-like structure as shown in Fig. 1c. PEP petals are approximately $0.8-1 \mu \mathrm{m}$ wide, $4-5 \mu \mathrm{m}$ long and 50-200 nm thick. 3,4-Dihydroxy-L-phenylalanine on the other hand, did not form any particular structure as can be noted from Fig. 1d. The absorbance and photo luminescent (PL) characteristics of the resulting structures, dispersed in ethanol, are shown in Fig. 1e-g. Two or more absorption bands are observed for all three nanoparticles. The PDA and LDA dispersions showed major absorption bands at $290 \mathrm{~nm}$ and $c a$. $430 \mathrm{~nm}$, whereas the PEP dispersion showed absorption bands at 290 and $c a .340 \mathrm{~nm}$ (Fig. 1e-g). These absorption bands can be attributed to the electronic transitions in various orbitals of $\mathrm{C}=\mathrm{C}, \mathrm{C}=\mathrm{O}$ and $\mathrm{C}-\mathrm{N}$, with the primary band at $c a .290 \mathrm{~nm}$ attributed to the red shifted pi-pi* orbital transition of aromatic $\mathrm{C}=\mathrm{C}$ bonds. ${ }^{25-28}$ The absorption spectrum acts as a guide to the primary excitation wavelength for the PL properties. Similar to the several other PL carbon dots and nanoparticle reported, the exact origins of the PL is unknown to us. ${ }^{25,29}$

The PL spectra were recorded at $290 \mathrm{~nm}$ excitation wavelength. When excited at $290 \mathrm{~nm}$, the corresponding fluorescent emission was recorded in the range $400-800 \mathrm{~nm}$, with the main emission band peaks at $c a .630 \mathrm{~nm}$. The second less intense peak at ca. $576 \mathrm{~nm}$ is due to the solvent background. Overall, the PL properties of structure formed from EP differ from that of DA and LDA significantly. The PL excitation spectra shown are for the $630 \mathrm{~nm}$ emission peak. These spectra demonstrate that the emission is also correlated to more than two electronic transition bands (identified from UV-Vis absorption spectra). This tells the complexity of these nanoparticle structures and the PL mechanism associated..$^{25}$ Simplistically, the origin of these PL properties can be attributed to the polycyclic aromatic domains in these particles as well as to the presence of amine groups in the monomers. ${ }^{30}$ Furthermore, it has been reported that the aggregation and morphology of polymeric materials can affect their absorption properties. ${ }^{31}$ However, these effects are not in the scope of present study.

It has been reported earlier that the concentration of precursor has a role in the size of resulting nanoparticles. ${ }^{10}$ In this study, the concentration of precursors was fixed at $3.3 \mathrm{mg}$ $\mathrm{mL}^{-1}$. It was found that increasing the quantity of base in the 
solution mixture resulted in smaller sized nanostructures (Fig. S1 $\dagger$ ), which is consistent with an earlier report by Xiong et al. ${ }^{22}$ In a separate experiment, adding the base drop by drop to the $\mathrm{DA} / \mathrm{H}_{2} \mathrm{O} / \mathrm{R}-\mathrm{OH}$ solution resulted in polydispersed PDA particles. This can be attributed to the dropwise addition of base under stirring which creates gradients of low and high alkaline microenvironments. Such an effect of alkaline microenvironment on the size of the PDA spheres can be utilized for producing hierarchically sized spheres ranging from nano to micron scales.

The dependence of PDA and PEP structure dimensions on base concentration was prominent for DA but less so for EP; LDA did not form any structure (see ESI, Fig. S1 $\dagger$ ). An extrapolation of the PDA size data obtained from various base concentrations (Fig. S2 $\dagger$ ), indicates that the lowest diameter of the PDA nanosphere that can be obtained with this method is approximately 30$50 \mathrm{~nm}$, which is comparable to carbon black spherical particles used as catalyst support for polymer electrolyte fuel cells. Further, a physical break-down of these nanospheres to quantum dot size will be of great interest to use them as biological probes. ${ }^{9,32}$

PDA was found to have a zeta potential $-40.1 \mathrm{mV}$, while PEP showed lower zeta potential at $-17.9 \mathrm{mV}$ (Fig. S3†). The lower negative charge in the zeta potential value of PEP indicates that the hydroxyl groups present in EP are less available at the surface. The differences in functional groups are correlated with the respective contact angles. The coverslips covered by cured epoxy without any particles, with PDA, and with PEP had a static water-air contact angle of $63^{\circ} \pm 2^{\circ}, 46^{\circ} \pm 3^{\circ}$, and $95^{\circ} \pm 3^{\circ}$ respectively (see Fig. S4 $\dagger$ ). The contact angle and zeta potential obtained for PDA agree with the values reported in the literature. ${ }^{17,33}$ The hydrophobic surface of PEP can be attributed to the presence of additional methyl group in the precursor EP and the lower surface concentration of amine and hydroxyl groups in the resulting PEP nanostructures, which is in agreement with the zeta potentials obtained. This is further supported by the NMR data. A comparison of ${ }^{1} \mathrm{H}$ NMR spectra of EP and PEP dissolved in DMSO-D6 (Fig. S5a $\dagger$ ) shows that all the peaks above $8.49 \mathrm{ppm}$, corresponding to the protons at amine and catechol groups, in EP were absent in PEP.

The hypothetical monomers for PDA and PEP are 5,6-dihydroxyindole and 2,3-dihydro-3,5,6-trihydroxy- $N$-methylindole respectively (Fig. S5b $\dagger$ ). Formation of 5,6-dihydroxyindole during the oxidative polymerization of dopamine is well documented. ${ }^{14,17}$ On the other hand, 2,3-dihydro-3,5,6-trihydroxy- $N$ methylindole (leuco-adrenochrome) is reported to be unstable. ${ }^{34}$ Instead, a zwitterionic compound isomerized under the influence of an alkali to form adrenolutin (3,5,6-trihydroxy$N$-methylindole) is predicted. ${ }^{34}$ The NMR for PEP provides no evidence of the formation of leuco-adrenochrome, or adrenolutin or an indole group. No broadening of the peaks representative of the protons in the benzene ring (6.4-6.8 ppm) was observed either. This indicates the absence of a polymeric structure with stronger bonds. Thus, the PEP petals formed can have weaker bonding, which is easily dissociated in DMSO-D6. The presence of an additional hydroxyl group in EP compared to DA would allow additional hydrogen bonded arrangements permitting extended 2D structures but with weaker interactions. Such a formation can also explain the melting of the petal structure at $600{ }^{\circ} \mathrm{C}$ during carbonization [Unpublished data] whereas PDA nanoparticle morphology is sustained at $1000{ }^{\circ} \mathrm{C}$ during carbonization. ${ }^{6}$

Fig. 2a and $\mathrm{b}$ depict the $2 \mathrm{D}$ excitation-emission spectra of PDA and PEP nanostructures obtained from laser scanning confocal microscopy (LSCM) setup. PDA nanoparticles show their fluorescence within the excitation wavelength of 520$590 \mathrm{~nm}$ whereas PEP microstructures revealed their fluorescence in the excitation range of $480-540 \mathrm{~nm}$. It can be noted that the excitation-emission wavelengths used are not the main bands recorded in the conventional spectrofluorometer (Fig. 1e and $\mathrm{f}$ ), but in compliance with the laser source and filters available in the LSCM. However, reasonable emission intensities were observed for the imaging purpose. Fluorescence images of PDA and PEP particles; which were dried from suspension, are shown in Fig. S6. $\dagger$

We exploit the fluorescence properties of these nanostructures to study the adsorption of PDA and PEP particles at the oil-water interface in emulsions by LSCM and crosscorrelate the stability and rheological behaviour of the particle-stabilized emulsions. Dodecane/water emulsions containing either spherical PDA or petal-like PEP particles were made using an identical procedure (see ESI $\dagger$ ) by suspending these particles in water, which is the continuous phase for the emulsions. The stability of these emulsions was examined via oil droplet size distribution measured over 15 days of aging, as shown in Fig. 3a and b. A continuously increasing droplet size for emulsions with PEP can be noted whereas PDA-stabilized emulsion trends toward a plateau after 15 days (Fig. S7†). The inferior stability of PEP containing emulsions is unexpected since PEP NP has a larger aspect ratio and a lower zeta potential, which induces less electrostatic repulsion from the negatively charged oil/water interface, compared with PDA particles. ${ }^{35}$

To examine the distribution of the PEP and PDA particles in the emulsions, 15 days aged samples were imaged via LSCM. The images reveal a nearly complete coverage of the oil droplets by PDA spherical particles, as seen in Fig. 3c. However, as shown in Fig. 3c (higher magnification image in Fig. S8†), PEP particles seem to form a self-aggregating phase rather than adsorb on the oil droplets. These images provide a direct observation of the adsorbing behaviour of PDA and PEP particles. The high coverage of oil droplets by PDA particles prevents the droplet coalescence whereas the poor coverage of oil droplets by PEP (a)

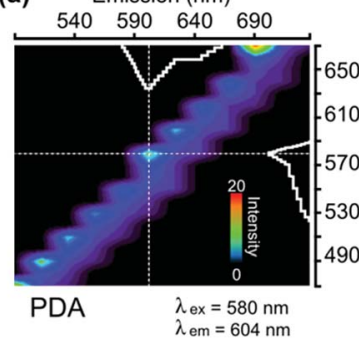

(b) Emission (nm) $\begin{array}{llll}540 & 590 & 640 \quad 690\end{array}$

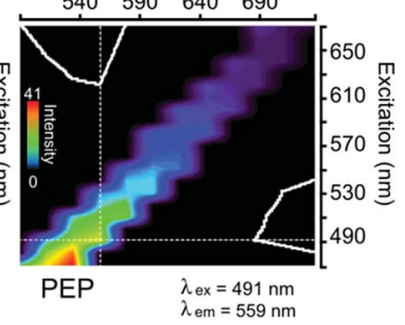

Fig. 2 Full excitation-emission spectra $(\Lambda-\lambda$ spectra) of different nanoparticles synthesized (a) polydopamine, (b) polyepinephrine, obtained using LSCM. 

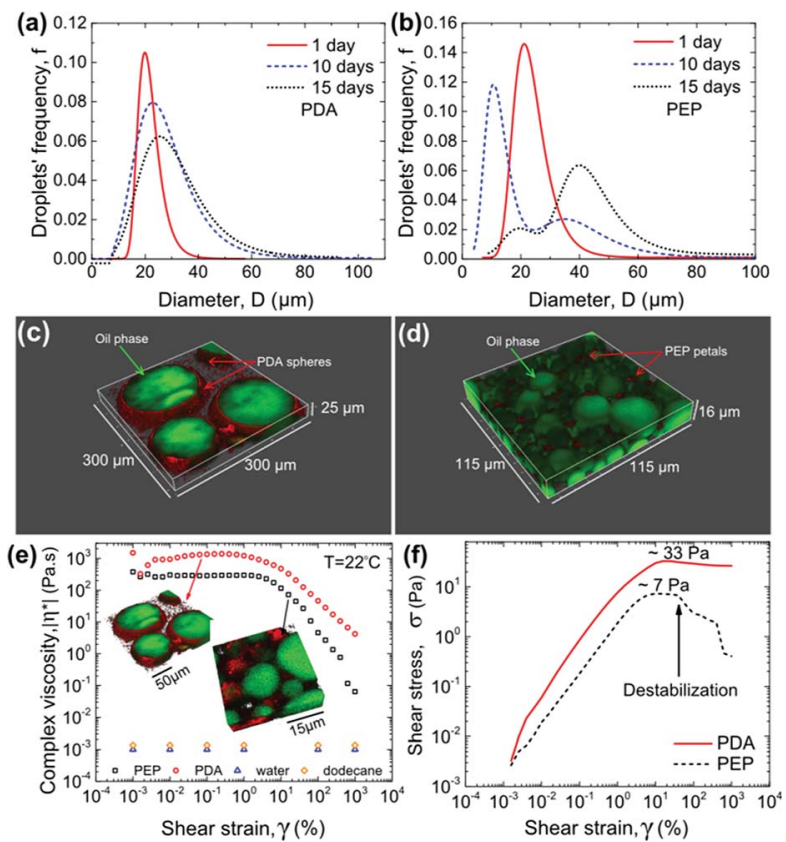

Fig. 3 Droplet size distributions of emulsions made with (a) PDA and (b) PEP; 3D confocal fluorescent images showing the distribution of (c) PDA and (d) PEP particles in the emulsion; (e) complex viscosity as a function of shear strain for emulsion with PDA (red circles) and PEP (black squares) after aging for 15 days; (f) shear stress as a function of shear strain for emulsion stabilized by PDA (solid line) and stabilized by PEP (dashed line).

does not help with the inhibition of droplet growth. A comparison of the fresh (1 day aged) and 15 days aged emulsions containing PEP particles (Fig. S8 $\dagger$ ) indicates that the PEP particles self-aggregated rather than move to the preferred region of oil/water interface. Whether it is due to particle size or surface properties of the PEP particles is not known. The corresponding rheological properties reveal a consistent behaviour (Fig. 3e); a baseline viscosity of water/dodecane emulsion could not be measured because of the rapid phase separation. The PDA-stabilized emulsion has a higher viscosity than the PEPcontaining emulsion (Fig. 3e). The poorer coverage of interface by PEP is less effective in inhibiting oil droplet growth and thereby resulting in lower viscosity compared to PDA-stabilized emulsions. The lower coverage of oil/water interface by PEP also results in the easier destabilization of the emulsion as revealed in the stress-strain response (Fig. 3f) for the two emulsions wherein a sudden decrease in the shear stress signal (showed by the arrow in Fig. 3f) at $\sim 7 \mathrm{~Pa}$ is observed when droplets undergo coalescence. On the other hand, PDA-stabilized droplets withstand the shear flow field imposed up to $\sim 33 \mathrm{~Pa}$. Our results indicate that although PEPs are more hydrophobic inherently and have larger aspect ratio, making them possibly a better candidate for stabilizing the oil droplets, the large size of PEPs and potentially stronger particle network formation in water phase jeopardizes their coverage of the oil-water interface. These results have opened up several interesting questions about the shape, size and wettability effects of nanoparticles employed for emulsion stability and further studies are warranted to fully understand the unexpected behaviour.

\section{Conclusions}

The results have shown that the fluorescence of particles, one with a commonly encountered spherical shape and another with an unusual shape can be a very powerful property for imaging studies to understand complex multiphase systems because they allow observation of how these particles organize/ adsorb at interfaces and self-aggregate. This study demonstrated intriguing fluorescent and structural properties from bio-inspired catecholamines DA, EP, and LDA as precursors. Applicability of these fluorescent nanostructures is multi-fold, for example in the realm of carbon-based nanotechnology. This study demonstrated one such imaging application for examining shape and wettability effect of particles on the behaviour of oil-in-water emulsions.

\section{Conflicts of interest}

There are no conflicts to declare.

\section{Acknowledgements}

Authors wish to thank Wade White and Pejman Ganjeh Anzabi (University of Calgary) for NMR measurements and discussion. V. O. K. and M. D. acknowledge Eyes-High Postdoctoral Scholarship from University of Calgary. M. T. acknowledges infrastructure funding from Canada Foundation for Innovation (CFI) and support from Natural Sciences and Engineering Research Council of Canada (NSERC) - Discovery Grant (DG) program. K. K. acknowledges partial support of this work from NSERC-DG and University of Calgary VPR-Seed Grant.

\section{Notes and references}

1 M. E. Gindy and R. K. Prud'homme, Expert Opin. Drug Delivery, 2009, 6, 865-878.

2 Y. Fang, D. Gu, Y. Zou, Z. Wu, F. Li, R. Che, Y. Deng, B. Tu and D. Zhao, Angew. Chem., Int. Ed., 2010, 49, 7987-7991.

3 Z. Yang, J. Ren, Z. Zhang, X. Chen, G. Guan, L. Qiu, Y. Zhang and H. Peng, Chem. Rev., 2015, 115, 5159-5223.

4 Q. Liu, Z. Pu, A. M. Asiri, A. O. Al-Youbi and X. Sun, Sens. Actuators, B, 2014, 191, 567-571.

5 H. Long, D. Del Frari, A. Martin, J. Didierjean, V. Ball, M. Michel and H. I. El Ahrach, J. Power Sources, 2016, 307, 569-577.

6 K. Ai, Y. Liu, C. Ruan, L. Lu and G. Lu, Adv. Mater., 2013, 25, 998-1003.

7 X. Zhang, S. Wang, L. Xu, L. Feng, Y. Ji, L. Tao, S. Li and Y. Wei, Nanoscale, 2012, 4, 5581.

8 X. Chen, Y. Yan, M. Müllner, M. P. Van Koeverden, K. F. Noi, W. Zhu and F. Caruso, Langmuir, 2014, 30, 2921-2925.

9 J.-H. Lin, C.-J. Yu, Y.-C. Yang and W.-L. Tseng, Phys. Chem. Chem. Phys., 2015, 17, 15124-15130.

10 J. Yan, L. Yang, M.-F. Lin, J. Ma, X. Lu and P. S. Lee, Small, 2013, 9, 596-603.

11 X. Jiang, Y. Wang and M. Li, Sci. Rep., 2014, 4, 1-6. 
12 S. Torquato and Y. Jiao, Nature, 2009, 460, 876-879.

13 R. Ni, A. P. Gantapara, J. de Graaf, R. van Roij and M. Dijkstra, Soft Matter, 2012, 8, 8826-8834.

14 T. G. Barclay, H. M. Hegab, S. R. Clarke and M. GinicMarkovic, Adv. Mater. Interfaces, 2017, 4, 1-38.

15 D. R. Dreyer, D. J. Miller, B. D. Freeman, D. R. Paul and C. W. Bielawski, Langmuir, 2012, 28, 6428-6435.

16 S. Hong, Y. S. Na, S. Choi, I. T. Song, W. Y. Kim and H. Lee, Adv. Funct. Mater., 2012, 22, 4711-4717.

17 H. Lee, S. M. Dellatore, W. M. Miller and P. B. Messersmith, Science, 2007, 318, 426-430.

18 M. Xiao, Y. Li, M. C. Allen, D. D. Deheyn, X. Yue, J. Zhao, N. C. Gianneschi, M. D. Shawkey and A. Dhinojwala, ACS Nano, 2015, 9, 5454-5460.

19 Z. F. Gao, T. T. Li, X. L. Xu, Y. Y. Liu, H. Q. Luo and N. B. Li, Biosens. Bioelectron., 2016, 83, 134-141.

20 A. H. Lu, G. P. Hao and Q. Sun, Angew. Chem., Int. Ed., 2011, 50, 9023-9025.

21 J. Liu, S. Z. Qiao, H. Liu, J. Chen, A. Orpe, D. Zhao and G. Q. Lu, Angew. Chem., Int. Ed., 2011, 50, 5947-5951.

22 S. Xiong, Y. Wang, J. Yu, L. Chen, J. Zhu and Z. Hu, J. Mater. Chem. A, 2014, 2, 7578-7587.

23 T. Yang, J. Liu, R. Zhou, Z. Chen, H. Xu, S. Z. Qiao and M. J. Monteiro, J. Mater. Chem. A, 2014, 2, 18139-18146.

24 Y.-P. Zhu, Y.-P. Liu and Z.-Y. Yuan, Chem. Commun., 2016, 52, 2118-2121.
25 D. Pan, J. Zhang, Z. Li and M. Wu, Adv. Mater., 2010, 22, 734738.

26 L. Lin and S. Zhang, Chem. Commun., 2012, 48, 10177.

27 S. Goswami, S. Chakraborty, A. K. Das, A. Manna, A. Bhattacharyya, C. K. Quah and H.-K. Fun, $R S C A d v$, 2014, 4, 20922-20926.

28 X. Dai, H. Wang, Z. Qian, Q. Yi, Y. Wang, S. Cong, J. Zhao, Y. Sun, J. Huang, J. Xiong, H. Luo and G. Zou, Appl. Phys. Lett., 2015, 107, 203108.

29 Y.-P. Sun, B. Zhou, Y. Lin, W. Wang, K. A. S. Fernando, P. Pathak, M. J. Meziani, B. A. Harruff, X. Wang, H. Wang, P. G. Luo, H. Yang, M. E. Kose, B. Chen, L. M. Veca and S.-Y. Xie, J. Am. Chem. Soc., 2006, 128, 7756-7757.

30 F. Ehrat, S. Bhattacharyya, J. Schneider, A. Löf, R. Wyrwich, A. L. Rogach, J. K. Stolarczyk, A. S. Urban and J. Feldmann, Nano Lett., 2017, 17, 7710-7716.

31 Y. Liu, J. Zhao, Z. Li, C. Mu, W. Ma, H. Hu, K. Jiang, H. Lin, H. Ade and H. Yan, Nat. Commun., 2014, 5, 5293.

32 G. E. LeCroy, S. K. Sonkar, F. Yang, L. M. Veca, P. Wang, K. N. Tackett, J.-J. Yu, E. Vasile, H. Qian, Y. Liu, P. (George) Luo and Y.-P. Sun, ACS Nano, 2014, 8, 4522-4529.

33 N. Nishizawa, A. Kawamura, M. Kohri, Y. Nakamura and S. Fujii, Polymers, 2016, 8, 62.

34 R. A. Heacock and B. D. Laidlaw, Nature, 1958, 182, 526-527. 35 B. Madivala, S. Vandebril, J. Fransaer and J. Vermant, Soft Matter, 2009, 5, 1717. 